\title{
Atg2A/B deficiency switches cytoprotective autophagy to non-canonical caspase-8 activation and apoptosis
}

\author{
Zhenyuan Tang ${ }^{1}$, Yoshinori Takahashi, ${ }^{* 1}$, Chong Chen ${ }^{1}$, Ying Liu ${ }^{2}$, Haiyan He ${ }^{1}$, Nikolaos Tsotakos ${ }^{1}$, Jacob M Serfass ${ }^{2}$, Melat T Gebru', \\ Han Chen ${ }^{3}$, Megan M Young ${ }^{1}$ and Hong-Gang Wang ${ }^{\star, 1,2}$
}

\begin{abstract}
Autophagosomal membranes are emerging as platforms for various cell survival and death signaling networks beyond autophagy. While autophagy-dependent cell death has been reported in response to a variety of stimuli, the underlying molecular mechanisms remain far from clear. Here, we demonstrate that inhibition of autophagosome completion by Atg2A/B deletion accumulates immature autophagosomal membranes that promote non-canonical caspase-8 activation in response to nutrient starvation via an intracellular death-inducing signaling complex (iDISC). Importantly, iDISC-induced caspase-8 dimerization and activation occurs on accumulated autophagosomal membranes and requires the LC3 conjugation machinery but is independent from the extrinsic pathway of apoptosis. Moreover, we have identified NF-kB signaling and c-FLIP as negative regulators of iDISC-mediated caspase8 activation and apoptosis. Collectively, these findings reveal autophagosomal membrane completion as a novel target to switch cytoprotective autophagy to apoptosis.
\end{abstract}

Cell Death and Differentiation (2017) 24, 2127-2138; doi:10.1038/cdd.2017.133; published online 11 August 2017

Macroautophagy (hereafter called autophagy) is a highly conserved intracellular lysosomal degradation process that is mediated by the formation of double-membrane vesicles called autophagosomes. Autophagy can be broken down into five major steps: initiation, nucleation of autophagosomal membranes (isolation membranes or phagophores), membrane elongation, closure, and autophagosome-lysosome fusion (formation of the autolysosome). ${ }^{1}$ The elongation step is accompanied by the conjugation of microtubule-associated protein 1 light chain 3 (LC3-I) to phosphatidylethanolamine (PE). The generation of LC3-PE (LC3-II) occurs through two ubiquitin-like conjugation reactions that covalently link the ubiquitin-like proteins Atg12 and LC3 to the substrates Atg5 and PE, respectively. LC3-II serves as a widely used marker of autophagosomes and also facilitates the selective recruitment of autophagic cargo by interaction with adapter proteins, such as p62. ${ }^{2}$ The sealing of autophagosomal membranes allows for fusion with late endosomes and/or lysosomes for cargo degradation. While the upstream machinery of autophagy is well-characterized, the precise molecular mechanism of autophagosome completion remains unknown. Yeast Atg2 has been identified as an important regulator of autophagosome completion., ${ }^{3,4}$ Similarly, loss of the two functionally redundant mammalian homologs, Atg2A and Atg2B, impairs autophagic flux and accumulates proteinase-K-sensitive immature autophagosomal membranes to suggest a conserved role in mammalian cells. ${ }^{5,6}$

The crosstalk between apoptosis and autophagy is highly complex and context dependent. ${ }^{7}$ Apoptosis is a strictly regulated cell death signaling pathway that is essential for organism development and homeostasis and can be initiated through extrinsic and intrinsic pathways. ${ }^{8,9}$ Activation of death receptors by death ligands triggers the Fas-associated protein with death domain (FADD)-dependent recruitment of procaspase-8 to the death-inducing signaling complex (DISC) for oligomerization and self-activation. ${ }^{10}$ In contrast, intrinsic activation induces Bax/Bak oligomerization and mitochondrial outer membrane permeabilization to release pro-apoptotic factors for procaspase- 9 activation. Caspase- 8 and caspase- 9 activate downstream executioner caspase-3/7 to initiate cell death. In addition, crosstalk between the extrinsic and intrinsic pathways exists via the caspase-8-mediated cleavage of $\mathrm{Bid}^{11}$ or caspase-3-mediated activation of caspase-8. ${ }^{12}$ While autophagy inhibits apoptosis to promote survival, autophagy has also been reported to facilitate cell death. ${ }^{7}$ However, the molecular signaling events that dictate whether autophagy suppresses or enhances cell death remain unclear.

We and others have recently found that autophagosomal membranes serve as platforms for an intracellular deathinducing signaling complex (iDISC) that activates caspase-8 to initiate the apoptotic cascade. ${ }^{13-22}$ Upon the formation of iDISC, procaspase-8 is recruited to the phagophore by two arms: (1) Atg12-Atg5: FADD: caspase-8; and (2) LC3-II: p62: caspase-8. Similar to its role in the canonical DISC, FADD serves as an adapter molecule for the recruitment of procaspase-8 to expanding phagophores via direct interaction with ATG5. ${ }^{13,14,17,19}$ Furthermore, p62 enhances canonical caspase- 8 activation by binding poly-ubiquitinated caspase- 8 at the DISC. ${ }^{23}$ Likewise, the interaction of p62 and LC3-II on the iDISC promotes procaspase- 8 recruitment and activation at phagophore membranes. ${ }^{13,16,18}$ Importantly, iDISCmediated apoptosis occurs independent from death receptor

\footnotetext{
${ }^{1}$ Department of Pediatrics, Penn State University College of Medicine, Hershey, PA, USA; ${ }^{2}$ Department of Pharmacology, Penn State University College of Medicine, Hershey, PA, USA and ${ }^{3}$ The Microscopy Imaging Facility, Penn State University College of Medicine, Hershey, PA, USA

${ }^{*}$ Corresponding author: H-G Wang or Y Takahashi, Department of Pediatrics, Penn State College of Medicine, 500 University Drive, H085, Hershey, PA 17033, USA. Tel: +1 717 531-4574 or +1 717 531-0003 x285882; E-mail: hwang3@ pennstatehealth.psu.edu or ytakahashi@pennstatehealth.psu.edu Received 13.3.17; revised 30.6.17; accepted 10.7.17; Edited by E Baehrecke; published online 11.8.17
} 
signaling and requires LC3-positive autophagic membranes, as the depletion of phagophores (3-MA treatment) or loss of the LC3 conjugation machinery (knockout of ATG5 or ATG7) suppresses iDISC-mediated apoptosis. ${ }^{13,15-17,19}$

In this study, we demonstrate that accumulation of immature autophagosomal membranes by the loss of Atg2A/B promotes iDISC-mediated, non-canonical caspase-8 activation and apoptosis. Furthermore, we identify NF-kB as a pro-survival factor that inhibits iDISC-mediated caspase-8 activation via the upregulation of c-FLIP. Collectively, these studies are the first to use a genetic approach to shift cytoprotective autophagy to iDISC-mediated apoptosis and identify autophagosome completion as a critical step that can redirect autophagy to apoptosis.

\section{Results}

Loss of Atg2A and Atg2B impairs autophagic flux and accumulates immature autophagosomal membranes. Atg2A and Atg2B have been suggested to be essential for the autophagosome completion step;,6 therefore, we first determined if depletion of Atg2A and Atg2B accumulates immature autophagosomal membranes to promote the iDISC assembly for the induction of apoptosis. To this end, CRISPR/ Cas9-mediated genome editing was employed to disrupt the expression of Atg2A and Atg2B (CrAtg2A/B) in THP-1 cells (Figure 1a), a pediatric acute myeloid leukemia (AML) cell line that is resistant to canonical caspase-8 activation due to the lack of DR4 and DR5 expression. ${ }^{24,25}$ Autophagic flux was assessed by culturing cells in complete or starvation (amino acid and serum-free) medium and in the presence or absence of the lysosomal inhibitor Bafilomycin A1 (BafA1) prior to immunoblotting for the autophagosome marker LC3-II and autophagic substrate $\mathrm{p} 62 .^{26}$ Consistent with previous reports, ${ }^{6}$ the loss of Atg2A/B significantly impairs basal and starvation-induced autophagic flux, as evident by the strong accumulation of p62 and LC3-II in CrAtg2A/B cells that is not further enhanced by BafA1 compared with wild-type cells (WT) (Figures $1 \mathrm{~b}$ and c). Autophagic flux was further assessed using tandem fluorescent-tagged LC3 (tfLC3; RFP-EGFP-LC3), in which differences in $\mathrm{pK}_{\mathrm{a}}$ result in the fluorescence quenching of EGFP but not RFP within the acidic environment of autolysosomes. ${ }^{26,27}$ Consistent with immunoblotting, RFP ${ }^{+} \mathrm{EGFP}^{+}$puncta (immature and completed autophagosomes) and $\mathrm{RFP}^{+} \mathrm{EGFP}^{-}$puncta (autolysosomes) are induced upon starvation in WT cells, indicating active autophagic flux (Figure 1d). In contrast, CrAtg2A/B cells strongly accumulate $\mathrm{RFP}^{+} \mathrm{EGFP}^{+}$puncta regardless of starvation to indicate a defect in autophagic degradation (Figure 1d).

To determine if Atg2A/B depletion impairs autophagosome completion, cells were starved and stained for a phagophore marker Atg16L1, which associates with Atg12-Atg5 at elongating phagophores and is released upon autophagosome completion. ${ }^{1,28}$ We found that CrAtg2A/B cells strongly accumulate p62-positive Atg16L1 puncta (Figure 1e) to indicate a defect in autophagosome completion. Next, we sought to directly visualize autophagosome structures by transmission electron microscopy (TEM). While electron- dense autolysosomal structures are abundant upon starvation of WT cells, CrAtg2A/B cells accumulate immature autophagosomal membranes that fail to be degraded (Figure 1f), which is consistent with a defect in autophagosome completion. Conclusively, these data indicate that the loss of Atg2A and Atg2B impairs autophagic flux and autophagosome completion to accumulate immature autophagosomal membranes.

Accumulation of immature autophagosomal membranes sensitizes cells to caspase-8-dependent apoptosis. Upon prolonged incubation in starvation medium, we found that Atg2A/B deficiency significantly reduces cell viability (Figure 2a). Consistent with iDISC-mediated apoptosis, starvation induces the cleavage and activation of caspase- 8 and caspase-3/7 in CrAtg2A/B cells (Figures $2 b-d$ ). If caspase-8 is the initiator caspase for apoptosis, inhibition or loss of caspase- 8 is expected to suppress caspase- $3 / 7$ activation. As expected, starvation-induced caspase-3/7 activity is blocked not only by the pan caspase inhibitor Z-VAD-FMK but also by the caspase-8 inhibitor Z-IETD-FMK to suggest caspase- 8 is an initiator caspase (Figure 2e). To further confirm the result, we knocked out caspase- 8 in CrAtg2A/B cells using CRISPR/Cas9 (Figure 2f). Caspase-8 depletion in CrAtg2A/B cells significantly suppresses the starvation-induced activation of caspase-3/7 to suggest that caspase-8 initiates the apoptotic cascade (Figure 2f). Of note, caspase-3/7 remains slightly activated in CrAtg2A/B cells lacking caspase-8 to suggest that the loss of autophagy may partially sensitize cells to mitochondrial apoptosis during starvation (Figure 2f); a result consistent with the cytoprotective role of autophagy during nutrient deprivation. ${ }^{29-31}$

To confirm that caspase- 8 is directly activated via the iDISC rather than a result of mitochondrial feedback upon starvation, we knocked out caspase-9 in CrAtg2A/B cells (Figure 2g). In support of the iDISC model, the loss of the intrinsic apoptotic pathway does not affect the initial activation of caspase-8 and caspase-3/7 in starved CrAtg2A/B cells (Figure $2 \mathrm{~h}$ ). However, at later time points, the loss of caspase- 9 partially suppresses caspase- 8 and caspase-3/7 activity in CrAtg2A/B cells to indicate that the initial activation of caspase-8 upon starvation is independent of intrinsic apoptotic pathway, but the mitochondrial feedback contributes to the full activation of caspase-8 and caspase-3/7 (Figure 2h). Next, we sought to determine if caspase- 8 is initiated independent of the extrinsic apoptosis pathway. As THP-1 cells are inherently resistant to TRAIL-induced apoptosis due to the lack of DR4 and DR5 expression (Supplementary Figure 1), we depleted Atg2A/B in TRAIL-sensitive HeLa cells (Supplementary Figure 2). Consistent with THP-1 cells, the loss of Atg2A/B sensitizes HeLa cells to starvation-induced cell death (Figure 2i). Importantly, the loss of Atg2A/B fails to further enhance TRAIL-induced apoptosis (Figure 2j), indicating that starvation-induced caspase-8 activation is initiated independent of the extrinsic apoptotic pathway. To determine if the phenotypes observed in CrAtg2A/B cells are due to the loss of Atg2A/B, CrAtg2A/B cells were transduced with lentiviruses encoding gRNAresistant GFP-Atg2A (GFP-Atg2 $A^{R}$ ). We found that the expression of GFP-Atg2 $A^{R}$ is sufficient for restoring the defect in p62 clearance (Figure 3a) and suppresses 

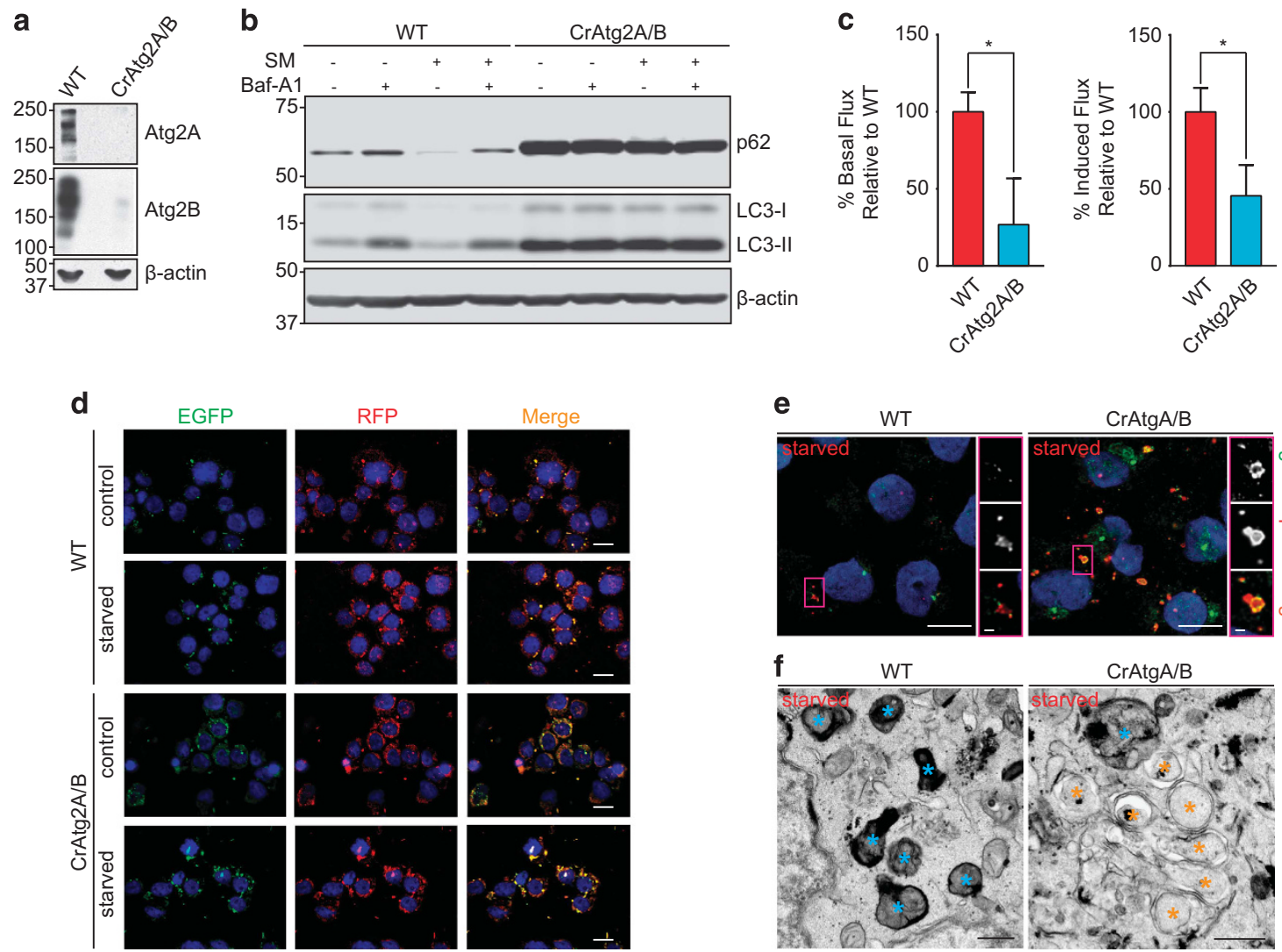

e
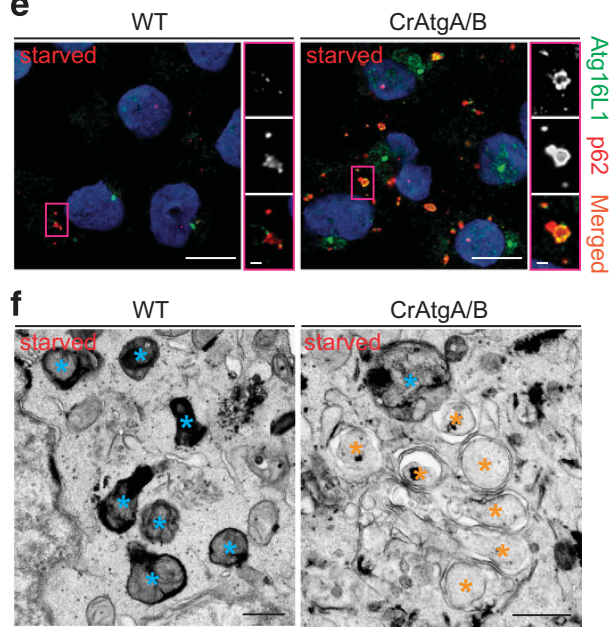

Figure 1 The loss of Atg2A and Atg2B impairs autophagic flux and results in the accumulation of immature autophagosomal membranes. (a) Atg2A and Atg2B (CrAtg2A/B) were targeted by the CRISPR/Cas9 genome editing system. Wild-type (WT) and Atg2A/B-deficient (CrAtg2A/B) THP-1 cells were subjected to immunoblotting using the indicated antibodies. (b) THP-1 cells were incubated in complete (CM) or starvation media (SM) for $2 \mathrm{~h}$ in the presence or absence of $100 \mathrm{nM}$ Bafilomycin A1 (BafA1) and subjected to immunoblotting using the indicated antibodies. (c) Basal and starvation-induced autophagic flux were quantified as described in the Methods. Mean \pm S.D. $(n=3)$, ${ }^{*} P<0.05$. (d) THP-1 cells stably expressing RFP-EGFP-LC3 were cultured in CM or SM for $2 \mathrm{~h}$, fixed and mounted with DAPI. Scale bars represent $20 \mu \mathrm{m}$. (e,f) THP-1 cells were cultured in SM for $2 \mathrm{~h}$ and subjected to (e) immunostaining using the indicated antibodies and mounted with DAPI. Scale bars represent $10 \mu \mathrm{m}, 1 \mu \mathrm{m}$ (insets); or (f) transmission electron microscopy. Asterisks represent autophagosomes (yellow) and autolysosomes (blue). Scale bars represent $0.5 \mu \mathrm{m}$

starvation-induced caspase-8 and caspase-3/7 activity (Figures $3 b$ and $c$ ) and apoptotic cell death (Figure $3 d$ ) in CrAtg2A/B cells. Collectively, these data indicate that the loss of Atg2A/B promotes starvation-induced activation of caspase-8 for the initiation of apoptosis.

The LC3 conjugation machinery is required for the noncanonical activation of caspase-8. Autophagy is an essential survival pathway for many cancer cells; thus, the inhibition of autophagic degradation may severely impair the cellular stress response to starvation to result in cell death. ${ }^{32}$ To determine if the enhanced caspase- 8 activation and apoptosis in CrAtg2A/B cells upon starvation is not simply due to the loss of autophagic degradation, we generated Atg7-deficient cells (CrAtg7) that are defective in LC3 conjugation (Figure 4a) and unable to assemble the platforms for iDISC assembly and procaspase-8 recruitment. Despite impaired autophagy, CrAtg7 cells fail to activate caspase- 8 or caspase- $3 / 7$ in response to starvation compared with CrAtg2A/B cells (Figures $4 \mathrm{~b}$ and $\mathrm{c}$ ). Moreover, the loss of Atg7 does not induce significant apoptosis upon starvation (Figure 4d). To further demonstrate that the LC3 conjugation machinery is required for starvation-induced caspase-8 activation in
CrAtg2A/B cells, we further knocked out Atg5 in CrAtg2A/B cells by CRISPR/Cas9 (Figure 4e). Indeed, the loss of Atg5 in CrAtg2A/B cells significantly impairs starvation-induced caspase-8 activation (Figure $4 \mathrm{e}$ ), indicating the indispensable role of Atg5 and/or LC3-II for iDISC assembly.

Our previous data show that procaspase- 8 can be recruited to the iDISC assembly sites via the Atg12-Atg5: FADD and p62: LC3-II arms. ${ }^{13}$ To determine if these two arms are functionally redundant, we generated FADD-deficient, p62-deficient, and FADD and p62 double-deficient CrAtg2A/B cells (Figure 4f). We found that starvation-induced caspase-8 activation is only marginally suppressed in CrAtg2A/B cells upon the loss of FADD or p62; however, depletion of both FADD and p62 in CrAtg2A/B cells significantly suppresses caspase-8 activation (Figure 4f; Supplementary Figure 3). Collectively, these results indicate that the two arms of iDISC are functionally redundant and the LC3 conjugation machinery is required for starvationinduced apoptosis in CrAtg2A/B THP-1 cells.

Loss of Atg2A/B promotes starvation-induced procaspase-8 dimerization on immature autophagosomal membranes. We next performed the bimolecular fluorescence complementation (BiFC) assay to investigate whether 
a

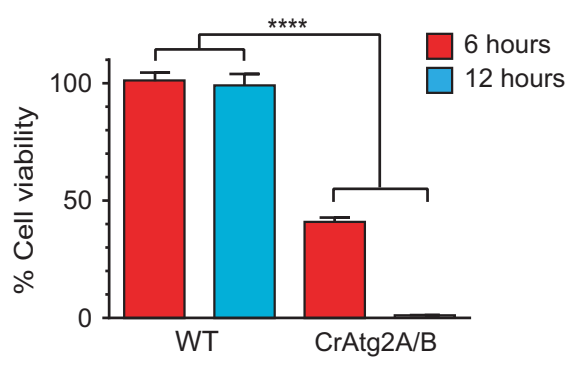

c
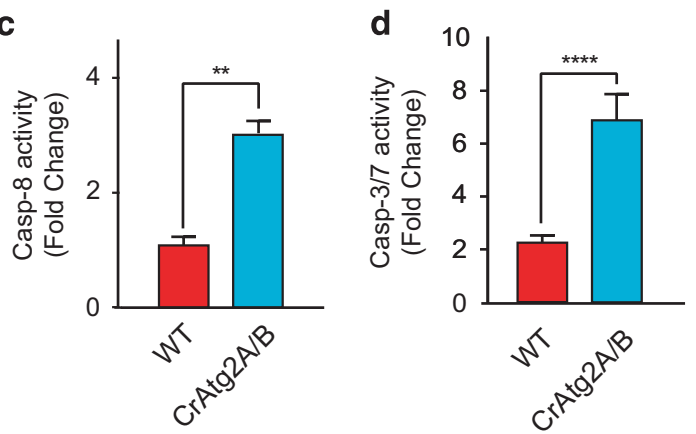

f
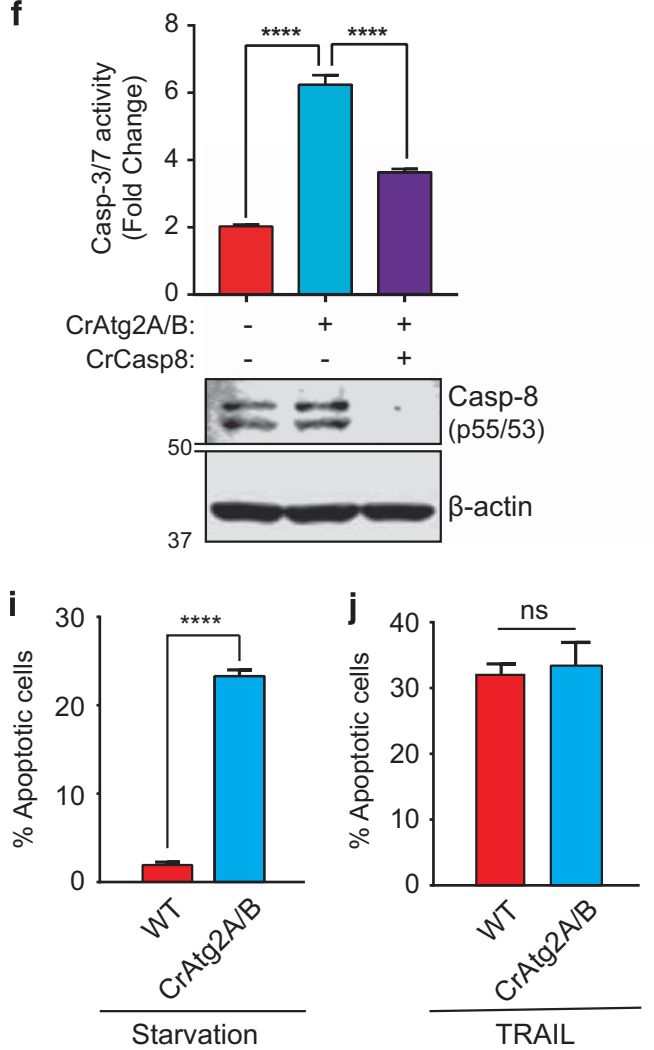

b

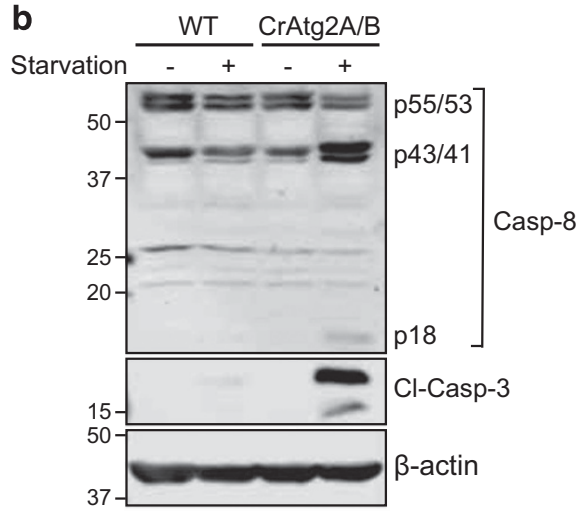

e

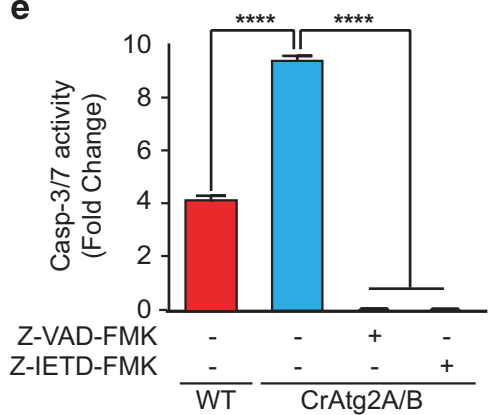

g
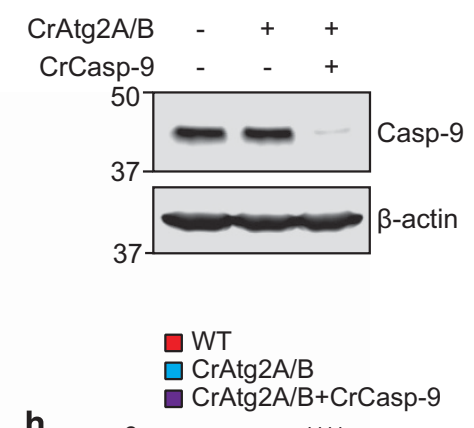

h
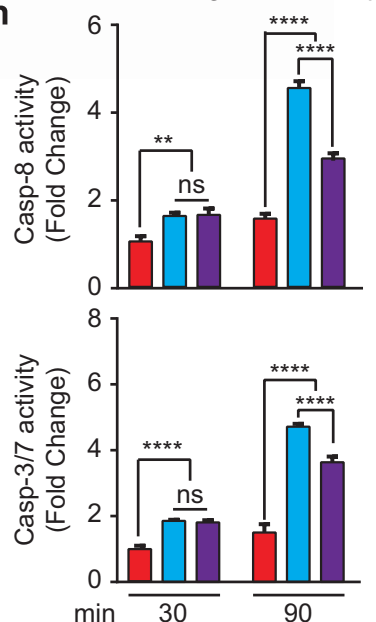
procaspase-8 dimerization occurs on immature autophagosomal membranes in CrAtg2A/B cells. The BiFC assay allows for the visualization of protein-protein interactions by the irreversible reconstitution of a full-length fluorescent protein. ${ }^{33}$ To evaluate caspase-8 dimerization in the absence of activation and cytotoxicity, the catalytically inactive caspase-8(C360A) mutant was fused to non-fluorescent N-terminal (VN155 (I152L)) and C-terminal (VC155) fragments of Venus. ${ }^{13}$ Fulllength fluorescent protein is generated by the reconstitution of VN155(I152L) and VC155 fragments upon caspase-8 dimerization. Consistent with the iDISC model, starvation-induced caspase-8(C360A) BiFC signals in CrAtg2A/B cells are positive for Atg16L1 and p62 (Figures 5a and b), indicating caspase-8 dimerization occurs on immature autophagosomal membranes. Moreover, caspase- 8 dimerization is significantly induced by starvation in Atg2A/B-deficient cells compared with non-starved conditions as well as starved WT cells (Figure $5 \mathrm{c}$ ). To more directly assess caspase-8 localization, we performed immunogold labeling of Venus protein for TEM analysis. Indeed, caspase-8(C360A) localizes to immature autophagosomal membranes in starved CrAtg2A/B cells (Figure $5 \mathrm{~d}$ ). To confirm that endogenous caspase- 8 is recruited to immature autophagosomal membranes for dimerization and activation, we established the in situ proximity ligation assay (PLA) using anti-p62 and anti-procaspase-8 antibodies. PLA is an antibody-based approach that detects endogenous protein interactions through the hybridization, rolling circle amplification, and fluorescence detection of complementary DNA strands. ${ }^{34}$ Consistent with BiFC and immunogold TEM, p62 and procaspase-8 PLA signals are selectively induced in

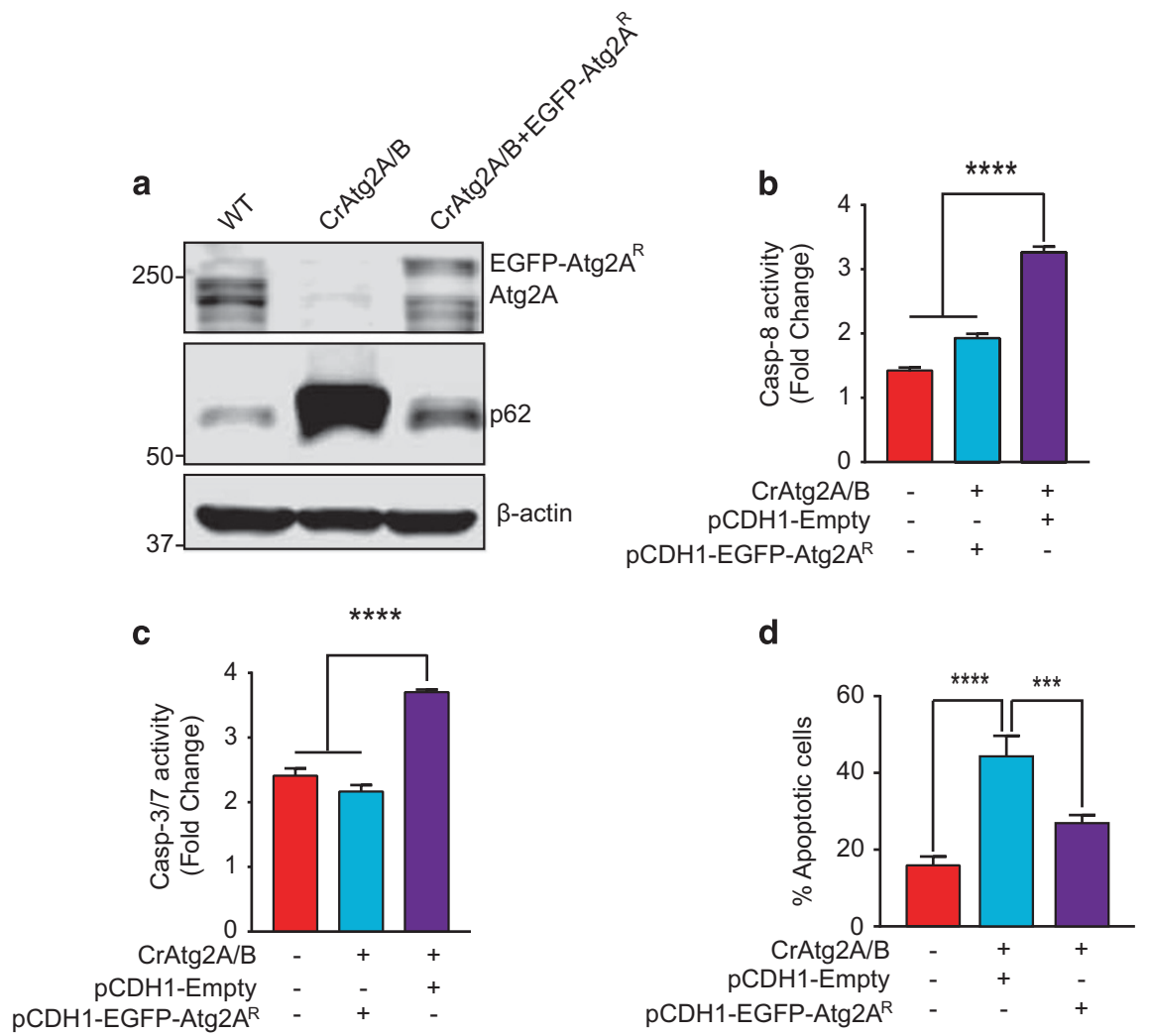

Figure 3 Expression of Atg2A restores autophagic flux and suppresses starvation-induced apoptosis in CrAtg2A/B cells. (a) CrAtg2A/B THP-1 cells were transduced with pCDH1-EGFP-Atg2 $A^{\mathrm{R}}$ and subjected to immunoblotting with the indicated antibodies. (b-d) WT, CrAtg2A/B and CrAtg2A/B+pCDH1-EGFP-Atg2 $A^{\mathrm{R}}$ THP- 1 cells were starved for $3 \mathrm{~h}$ and subjected to (b) caspase-8 activity assays and (c) caspase-3/7 activity assays; or (d) starved for $6 \mathrm{~h}$ and subjected to Annexin-V and 7-AAD staining flow cytometry analyses. All values are mean \pm S.D. $(n=3) .{ }^{* \star *} P<0.001,{ }^{* \star \star *} P<0.0001$

Figure 2 Accumulation of immature autophagosomal membranes sensitizes cells to caspase-8-dependent apoptosis. (a) THP-1 cells were incubated in CM or SM for $6 \mathrm{~h}$ and $12 \mathrm{~h}$ and subjected to cell viability assays. (b) THP-1 cells were incubated in CM or SM for $6 \mathrm{~h}$ and subjected to immunoblotting using the indicated antibodies. (c,d) THP-1 cells were starved for $3 \mathrm{~h}$ and subjected to (c) caspase-8 activity assays and (d) caspase-3/7 activity assays. (e) THP-1 cells were incubated in CM or SM for $6 \mathrm{~h}$ in the presence or absence of $50 \mu \mathrm{M}$ of Z-VAD-FMK or Z-IETD-FMK and subjected to caspase-3/7 activity assays. (f) CrAtg2A/B+CrCasp8 THP-1 cells were generated by CRISPR/Cas9 and subjected to immunoblotting with the indicated antibodies. WT, CrAtg2A/B and CrAtg2A/B+CrCasp8 THP-1 cells were incubated in SM for $3 \mathrm{~h}$ and subjected to caspase-3/7 activity assays. (g) CrAtg2A/B+CrCasp-9 THP-1 cells were generated by CRISPR/Cas9 and subjected to immunoblotting with the indicated antibodies. (h) WT, CrAtg2A/B and CrAtg2A/B+CrCasp-9 THP-1 cells were incubated in SM for 30 or 90 min and subjected to caspase-8 and caspase-3/7 assays. (i,j) WT and CrAtg2A/B HeLa cells were incubated in SM (i) or treated with $100 \mathrm{ng} / \mathrm{ml}$ TRAlL for $6 \mathrm{~h}$ (j) and subjected to Annexin-V and 7-AAD staining flow cytometry analyses. ${ }^{* *} P<0.01,{ }^{* * \star} P<0.001,{ }^{* * * *} P<0.0001$ 


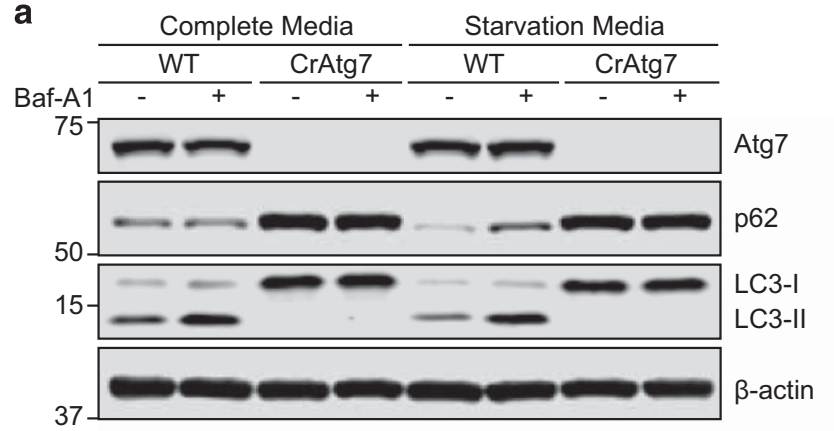

d

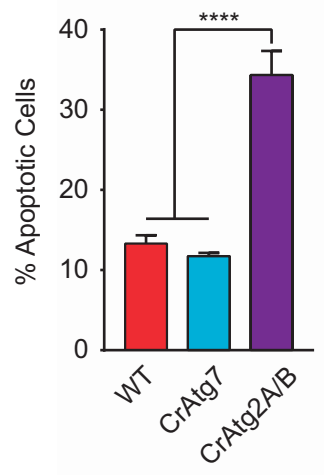

b

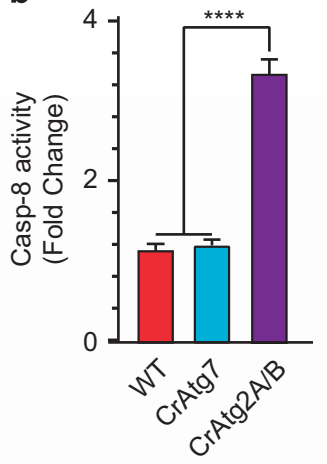

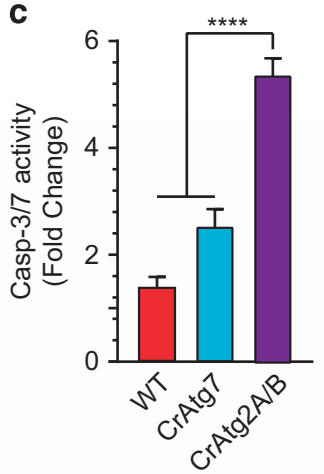

f

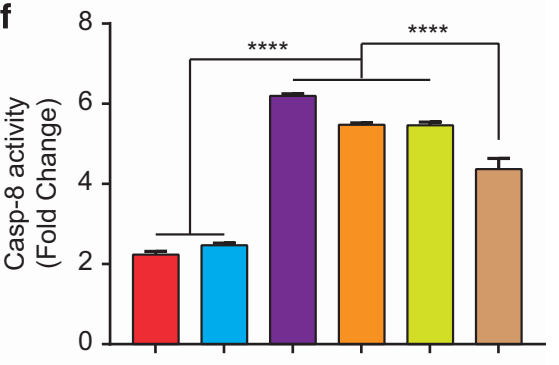

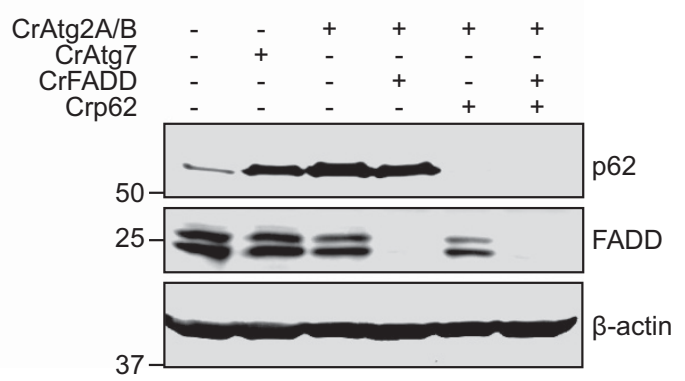

Figure 4 Non-canonical activation of caspase-8 requires the LC3 conjugation machinery. (a) Atg7 was targeted using CRISPR/Cas9 genome editing. WT and Atg7-deficient (CrAtg7) THP-1 cells were incubated in CM or SM for $2 \mathrm{~h}$ in the presence or absence of $100 \mathrm{nM} \mathrm{BafA1}$ and subjected to immunoblotting using the indicated antibodies. (b-d) WT, CrAtg7 and CrAtg2A/B THP-1 cells were incubated in SM for $3 \mathrm{~h}$ and subjected to (b) caspase-8 activity assays and (c) caspase-3/7 activity assays; or (d) for $6 \mathrm{~h}$ and subjected to Annexin-V and 7-AAD staining and flow cytometry analyses. (e,f) CRISPR/Cas9 was used to generate (e) CrAtg2A/B+CrAtg5 THP-1 cells or (f) $\mathrm{CrAtg} 2 A / B+C r F A D D, C r A t g 2 A / B$ + Crp62, CrAtg2A/B+CrFADD+Crp62 and cells were starved for $3 \mathrm{~h}$ and subjected to caspase-8 activity assays. All values are mean \pm S.D. $(n=3)$. ${ }^{* * \star} P<0.0001$

starved CrAtg2A/B cells and colocalize with the phagophore marker mCherry-Atg5 (Figure 5e). Taken together, these data indicate that starvation induces the self-association of caspase-8 on immature autophagosomal membranes in CrAtg2A/B cells.

Starvation promotes caspase-8 activation by suppression of NF-KB signaling and downregulation of c-FLIP. While CrAtg2A/B cells strongly accumulate p62 and immature autophagosomal membranes under basal and starvation conditions, cells only activate caspase- 8 and initiate apoptosis when starved. In addition to its autophagic and apoptotic functions, p62 promotes NF-kB signaling for cell survival, ${ }^{35-37}$ and p62 accumulation in response to autophagy inhibition is sufficient to activate NF-kB signaling. ${ }^{38,39}$ As NF-KB directly regulates the expression of c-FLIP (cellular FLICE-inhibitory protein), a well-established inhibitor of caspase-8, ${ }^{40-43}$ we hypothesized that the accumulation of $p 62$ in CrAtg2A/B cells promotes NF-kB signaling and c-FLIP expression to limit iDISC-mediated caspase-8 activation under basal conditions.
While the C-FLIP isoform $C$-FLIP $\mathrm{P}_{\mathrm{L}}$ limits the full activation of

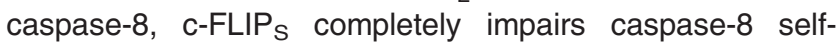
processing and activation. ${ }^{41}$ Consistent with our hypothesis,

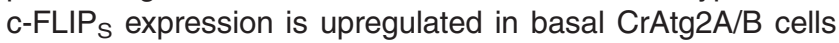
but suppressed upon starvation (Figure 6a). Moreover, starvation impairs NF-kB signaling, as evident by a decrease in the nuclear translocation of p65/RelA (Figure 6b). To determine whether c-FLIP directly interacts with caspase-8 on immature autophagosomal membranes, we transiently expressed caspase-8(C360A)-VC155 and c-FLIP-VN155 (I152L) in WT and CrAtg2A/B cells and stained for Atg16L1 (Figure 6c). As expected, c-FLIP and caspase-8 BiFC signals are only observed in CrAtg2A/B cells and colocalize with Atg16L1-positive immature autophagosome membranes (Figure 6c). Next, we sought to directly investigate the role of c-FLIP in iDISC-mediated apoptosis by siRNA knockdown. Transient loss of c-FLIP under nutrient-rich conditions significantly induces caspase-8 activation in CrAtg2A/B cells compared with WT cells to suggest that the downregulation of c-FLIP triggers iDISC-mediated apoptosis in 
a

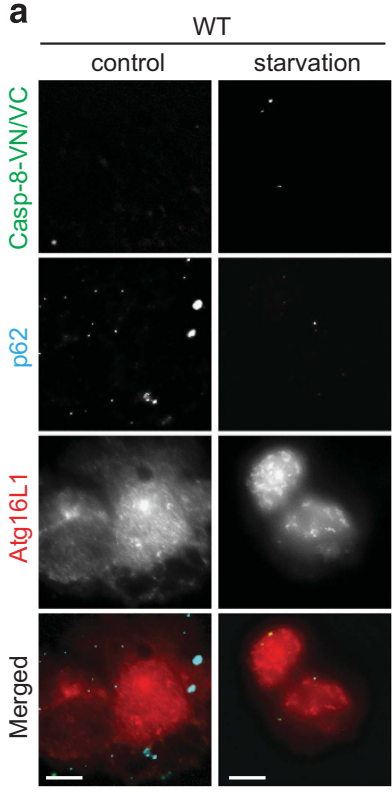

b
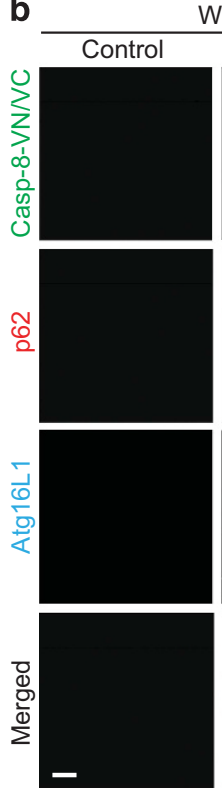

WT
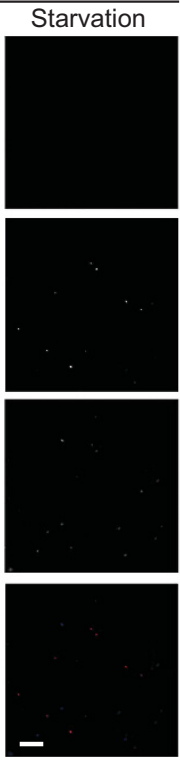
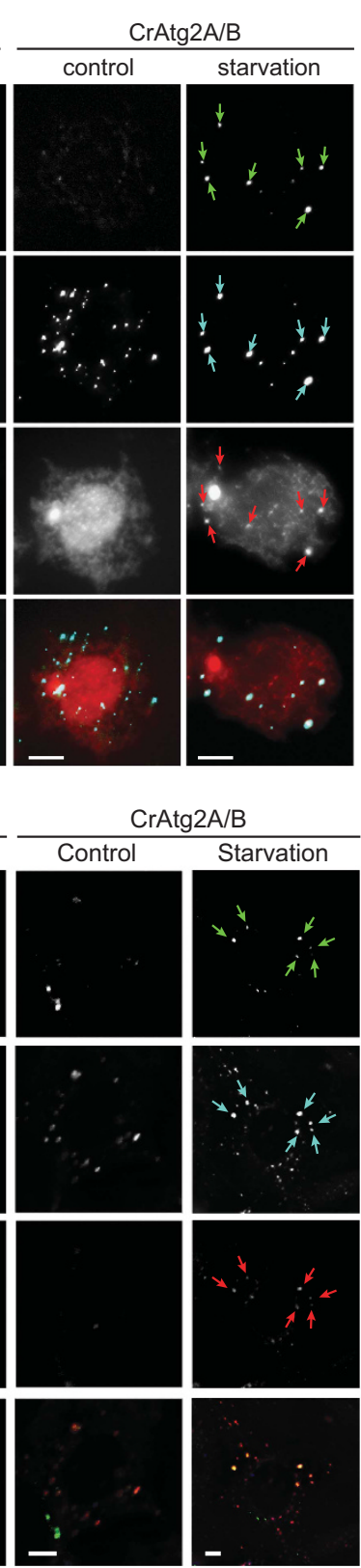

d

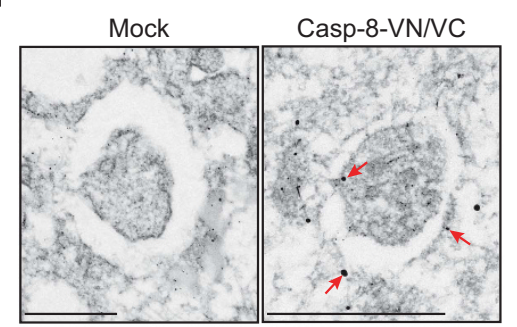

e

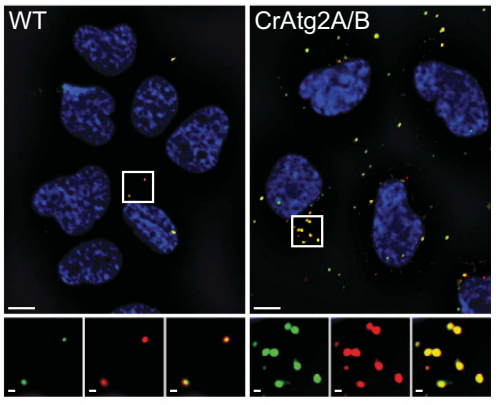

Figure 5 Loss of Atg2A/B promotes procaspase-8 dimerization on immature autophagosomal membranes. (a,b) Caspase-8(C360A)-VN155(I152L) and Caspase-8(C360A)VC155 (Casp-8-VN/VC) were transfected into WT and CrAtg2A/B (a) THP-1 (2 h starvation) or (b) U-2 OS cells (1.5 h starvation) and subjected to immunostaining using the indicated antibodies. Scale bars, $10 \mu \mathrm{m}$. (c) U-2 OS WT and CrAtg2A/B cells were transfected with Casp-8-VN/VC and incubated in CM or SM for $1.5 \mathrm{~h}$ and subjected to immunofluorescence imaging. BiFC signal fluorescence intensity is presented as the mean \pm S.D. ( $n=62,68,59$ and 52 cells, respectively). ns, non-significant, ${ }^{* * *} P<0.001$. (d) U-2 OS CrAtg2A/B cells were transfected with or without Casp-8-VN/VC and incubated in SM for $3 \mathrm{~h}$ before immunogold TEM using anti-GFP labeling of Venus. Arrows indicate gold particles on autophagosomal membranes. Scale bars, $1 \mu \mathrm{m}$. (e) U-2 OS cells were transduced with mCherry-Atg5 and starved for $2 \mathrm{~h}$ prior to proximity ligation assay (PLA) with guinea pig anti-p62 and rabbit anti-caspase-8 (full-length specific) antibodies. Scale bars, $10 \mu \mathrm{m}, 1 \mu \mathrm{m}$ (e insets) and $2 \mu \mathrm{m}$ (d insets)

CrAtg2A/B cells (Figures $6 \mathrm{~d}$ and e). To determine whether NF-KB directly modulates iDISC-mediated apoptosis, we treated WT, CrAtg2A/B, and CrAtg7 cells with the selective IKK inhibitor BMS-345541 under nutrient-rich conditions. While BMS-345541 downregulates c-FLIP expression in both WT and CrAtg2A/B cells (Figure 6f), the induction of apoptosis is only observed in CrAtg2A/B cells but not autophagy-competent WT cells or iDISC-deficient CrAtg7 cells (Figure 6g). Moreover, BMS-345541 selectively induces the time-dependent activation of caspase-8 in CrAtg2A/B cells but not WT or CrAtg7 cells (Figure 6h), suggesting that the accumulation of immature autophagosomal membranes in CrAtg2A/B cells 'primes' cells for iDISC-mediated caspase-8 activation. Conclusively, our data demonstrate that pro-survival NF-KB signaling promotes C-FLIP expression to limit iDISC-mediated caspase-8 activation in CrAtg2A/B cells under nutrient-rich conditions. 


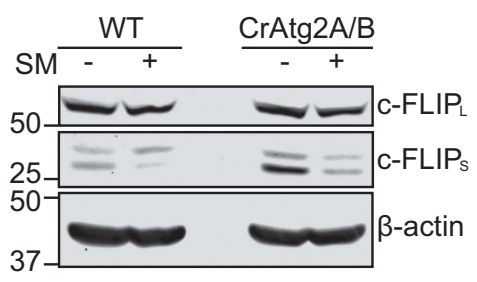

C

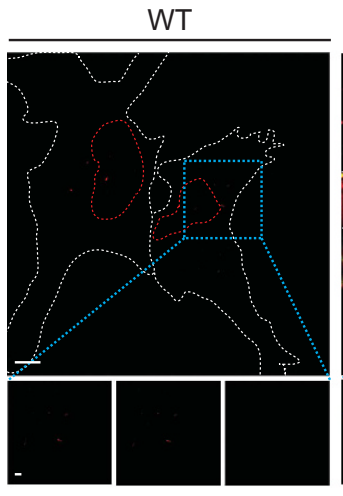

Merge Atg16L1 Casp8-VC Merge Atg16L1 Casp8-VC \& cFLIP-VN

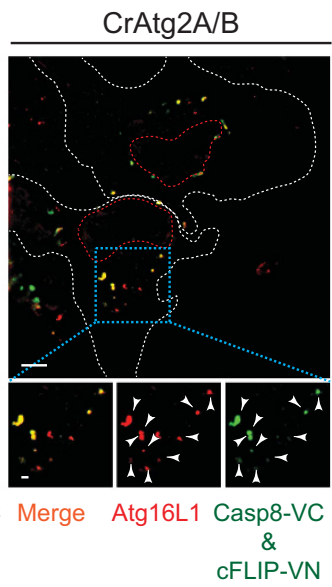

b
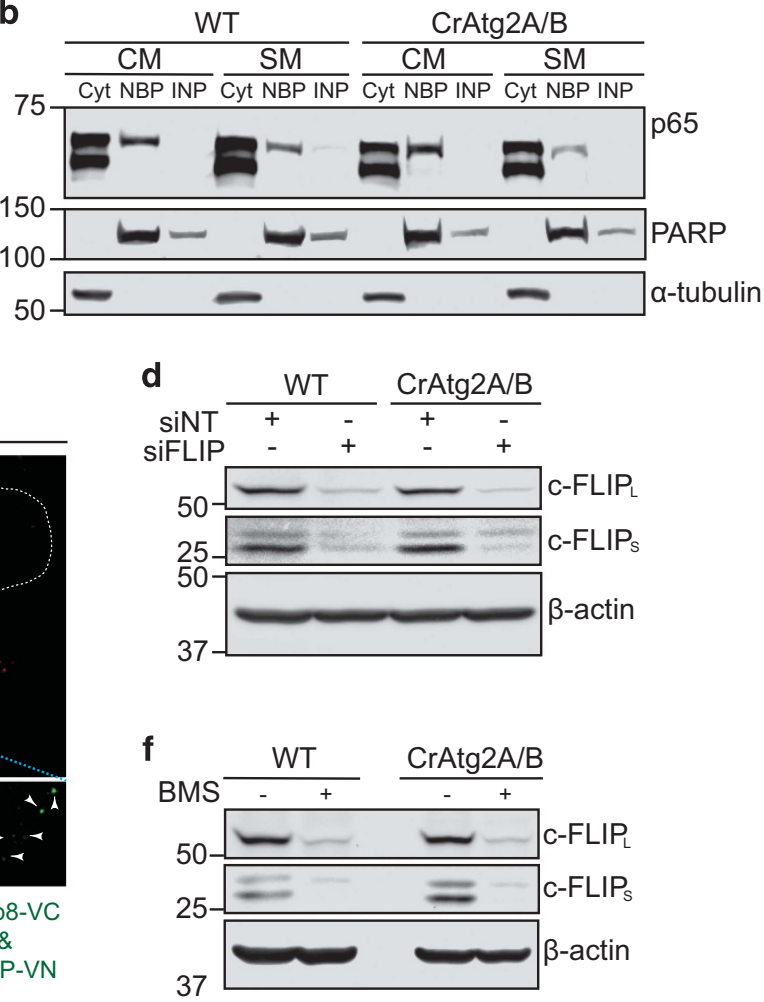

37 e

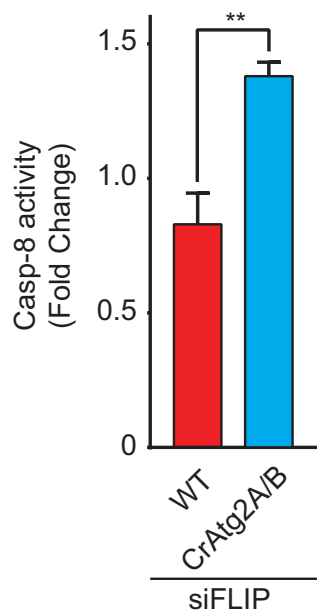

g

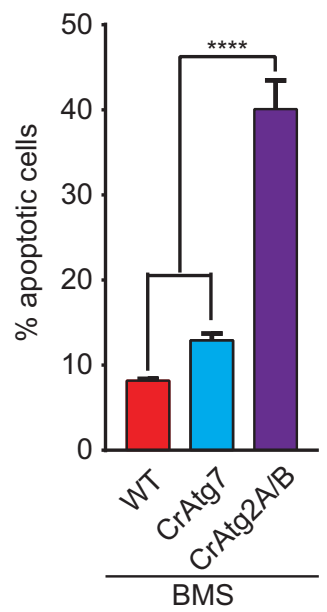

h 3.5

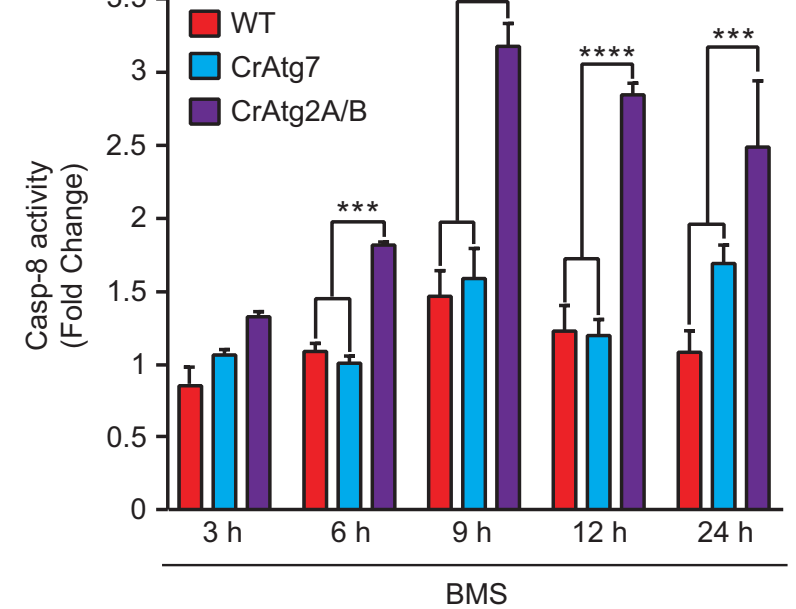

Figure 6 NF-KB and C-FLIP suppress iDISC-mediated caspase-8 activation. (a) WT and CrAtg2A/B THP-1 cells were incubated in CM or SM for $2 \mathrm{~h}$ and subjected to immunoblotting with the indicated antibodies. (b) WT and CrAtg2A/B THP-1 cells were incubated in CM or SM for $2 \mathrm{~h}$ and subjected to nuclear fractionation and immunoblotting with the indicated antibodies (Cyt, cytoplasmic; NBP, nuclear binding protein; INP, insoluble nuclear proteins). (c) C-FLIP-VN155(I152L) and Caspase-8(C360A)-VC155 were transfected into WTand CrAtg2A/B U-2 OS cells for $24 \mathrm{~h}$ prior to Atg16L1 immunostaining and fluorescence microscopy analyses. Scale bars, $10 \mu \mathrm{m}, 2 \mu \mathrm{m}$ (insets). (d,e) WTand CrAtg2A/B THP-1 cells were transfected with non-targeting (siNT) or c-FLIP siRNA and subjected to (d) immunoblotting after $24 \mathrm{~h}$ with the indicated antibodies; or (e) caspase-8 assays after $72 \mathrm{~h}$. (f) WT and CrAtg2A/B THP-1 cells were treated with or without $6.25 \mu \mathrm{M}$ BMS-345541 (BMS) for $12 \mathrm{~h}$ and subjected to immunoblotting with the indicated antibodies. (g,h) WT, CrAtg7 and CrAtg2A/B THP-1 cells were treated with $6.25 \mu \mathrm{M}$ BMS-345541 (g) for $12 \mathrm{~h}$ and subjected to Annexin-V and 7-AAD staining followed by flow cytometry analyses; or (h) for $3,6,9,12$ and $24 \mathrm{~h}$ and subjected to caspase-8 activity assays. All values are mean \pm S.D. $(n=3) .{ }^{\star \star} P<0.01,{ }^{* \star *} P<0.001,{ }^{\star \star \star \star \star} P<0.0001$

\section{Discussion}

Here, we demonstrate a molecular mechanism by which cytoprotective autophagy is switched to apoptosis through the non-canonical, iDISC-dependent activation of caspase-8 on immature autophagosomal membranes (Figure 7). First, we have shown that the loss of Atg2A/B leads to the accumulation of immature autophagosomal membranes by impairing autophagosome completion. Notably, starvation induces the dimerization of procaspase-8 on immature autophagosomal 


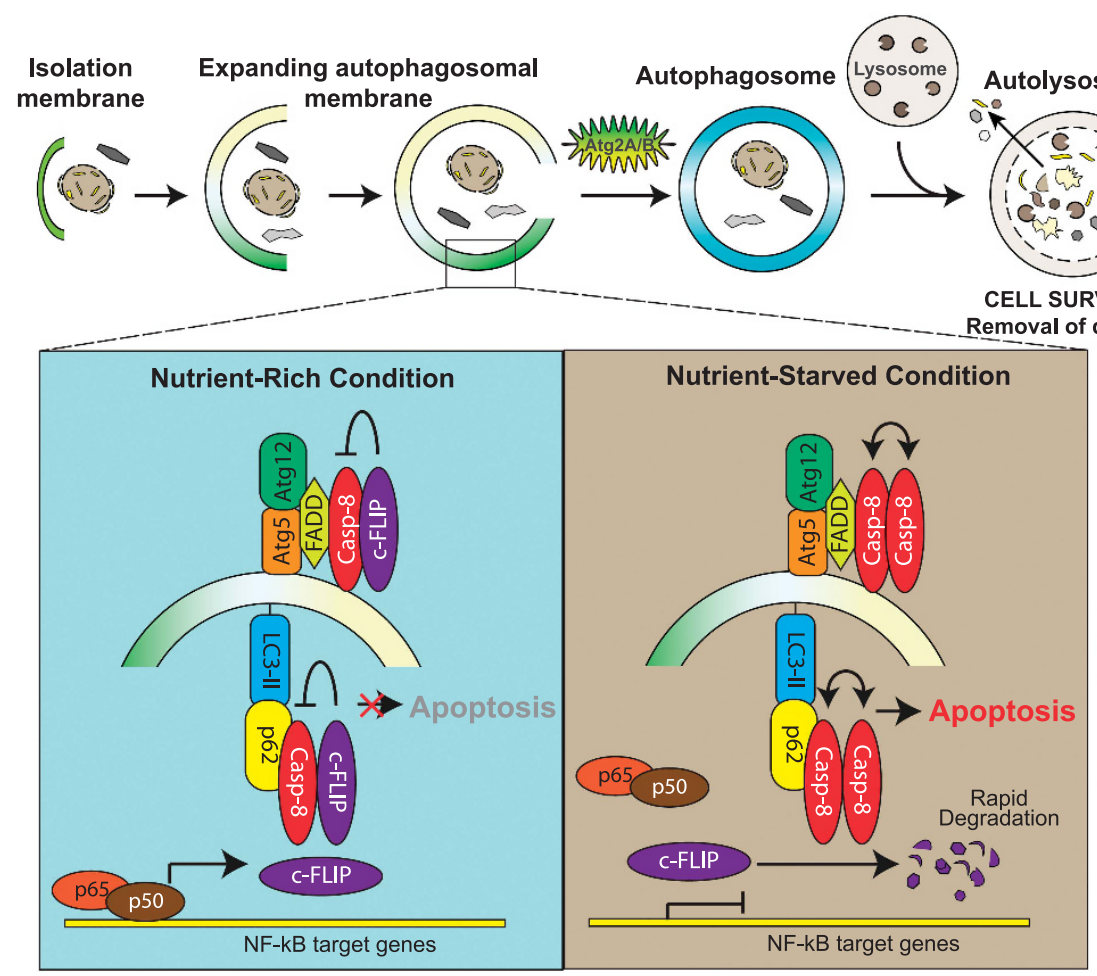

Figure 7 Proposed molecular model by which the inhibition of autophagosome completion promotes iDISC assembly for the non-canonical activation of caspase-8. During autophagy, autophagosomal membranes elongate, close and eventually fuse with lysosomes for the cytoprotective degradation of cargo. Inhibition of autophagosome completion by the loss of Atg2A/B promotes the formation and accumulation of LC3-II: p62: caspase-8 and Atg12-Atg5: FADD: caspase-8 complexes (iDISCs) on immature autophagosomal membranes. NF-kB activation upregulates the caspase-8 inhibitor c-FLIP to suppress caspase-8 activation under nutrient-rich conditions. Nutrient starvation suppresses NF-kB nuclear translocation, resulting in c-FLIP downregulation and the non-canonical activation of caspase-8 at autophagosomal membranes for the initiation of apoptosis

membranes in CrAtg2A/B cells to initiate the apoptotic cascade in a manner that is independent of canonical apoptosis pathways. Moreover, caspase activation requires the LC3 conjugation machinery to demonstrate that autophagic membranes can promote the non-canonical activation of caspase-8. Finally, our data reveal that NF- $\mathrm{kB}$ signaling serves as a pro-survival pathway to limit basal caspase-8 activation in CrAtg2A/B cells via the upregulation of c-FLIP. Suppression of $\mathrm{NF}-\mathrm{kB}$ signaling by nutrient starvation or pharmacological inhibition results in the rapid downregulation of short-lived c-FLIP to allow for iDISC-mediated activation of caspase-8 in CrAtg2A/B cells.

Autophagy promotes tumor growth by providing energy and building blocks that are required to support the proliferative and metabolic demands in the tumor microenvironment. High autophagic flux is associated with poor patient prognosis, enhanced metastasis, and resistance to chemotherapy. ${ }^{44}$ As a result, over a dozen clinical trials have investigated the use of two FDA-approved lysosomal inhibitors, chloroquine and hydroxychloroquine, as a mechanism to impair autophagic flux and enhance chemotherapeutic efficacy. ${ }^{45}$ Despite being well-tolerated and inhibiting autophagy in vivo, only moderate to marginal improvements in efficacy have been reported. ${ }^{45}$ Moreover, chloroquine is a lysosomotropic agent that has autophagy-independent effects. Indeed, chloroquine promotes cell death in both autophagy-competent and -deficient cells (Supplementary Figure 4). Our results suggest that selectively blocking autophagic flux by inhibition of autophagosome completion serves as a novel strategy to take advantage of the high pro-survival autophagic flux of cancer cells for iDISC-mediated apoptosis. Therefore, autophagosome completion is an attractive target for the future development of autophagy inhibitors for cancer therapy.

While we propose that inhibition of autophagosomal membrane closure is a novel approach for cancer therapy, our limited knowledge of the molecular mechanism of autophagosome completion presents a barrier for translation. In fact, there are currently no selective pharmacological inhibitors that block autophagy at the completion step. The ER-resident protein vacuole membrane protein 1 (VMP-1) presents an alternative gene target for autophagosome completion, as VMP-1 depletion promotes the accumulation of phagophores. ${ }^{5} \mathrm{~A}$ better understanding of the signaling networks regulating phagophore closure will allow for the development of more selective inhibitors of autophagosome completion. However, current progress in understanding the molecular mechanism is hindered by the lack of appropriate high-content assays that can distinguish open and closed autophagosomal structures. Therefore, further assay development is also needed.

We have also revealed that the accumulation of immature autophagosomal membranes upregulates c-FLIP through NF-kB signaling to limit iDISC-mediated caspase- 8 activation and apoptosis. It has been shown that p62 promotes TRAF6 oligomerization and polyubiquitination for IKK and NF-kB activation. $^{35-37}$ As TRAF6 is decreased in response to the 
iDISC-inducer bortezomib to coincide with autophagydependent apoptosis, ${ }^{46}$ we propose that TRAF6 may mediate NF-kB activation in response to 62 accumulation in CrAtg2A/ $B$ cells to suppress iDISC-mediated apoptosis. Furthermore, while knockdown of c-FLIP promotes significant caspase-8 activation in CrAtg2A/B cells, inhibition of NF-KB results in a more robust activation of caspase- 8 that mimics starvation, suggesting that NF-KB may drive the expression of additional unknown regulators of iDISC activation. Ultimately, combination of inhibitors of autophagosome completion and NF-kB signaling may be necessary to robustly induce iDISC-mediated apoptosis for cancer therapy.

Interestingly, in addition to the initiation of apoptosis, phagophores are emerging as unique signaling platforms for necroptosis and MAPK/ERK signaling. ${ }^{47,48}$ In fact, the Atg5and p62-dependent recruitment of necrosome components (RIPK1, RIPK3 and MLKL) to phagophores has been reported to be critical for the activation of necroptosis. ${ }^{47-49}$ As caspase8 can directly suppress the activation of necroptosis via the cleavage and inactivation of RIPK $1,{ }^{50}$ elucidating the crosstalk between iDISC and necrosome signaling on autophagosomal membranes is of great interest. Moreover, we propose that inhibition of autophagy at the autophagosome completion step by the loss of Atg2A/B will allow for the further investigation of autophagosomal membranes as dynamic cellular signaling platforms beyond serving as a means for autophagic cargo degradation.

Collectively, we have identified the last step of autophagosome formation as a novel target to switch cytoprotective autophagy to apoptosis through the non-canonical activation of caspase-8 on immature autophagosomal membranes. While the biological relevance of iDISC-mediated cell death under physiological conditions has not been established, several studies have demonstrated the importance of key autophagy genes (e.g., Atg5, Atg7) in programmed cell death during the development of Arabidopsis thaliana and Drosophila melanogaster ${ }^{51-54}$ The fact that both the LC3 conjugation machinery and caspase activation are required for developmental programmed cell death in these organisms suggests that iDISC-mediated apoptosis may have a physiological role and this will require further investigation. Nevertheless, targeting autophagosome completion for the initiation of iDISC-mediated apoptosis provides a timely and novel chemotherapeutic strategy to redirect cytoprotective autophagy to cell death in cancer cells that frequently display high autophagic flux and resistance to canonical apoptotic signaling.

\footnotetext{
Materials and Methods

Reagents, antibodies and plasmids. The chemicals used in this work are: Bafilomycin A1 (LC Laboratories, Woburn, MA, USA, B-1080); BMS-345541 (Sigma, St. Louis, MO, USA, B9935); TRAIL (VWR, Radnor, PA, USA, 10787-196). The following primary antibodies have been used in this work: rabbit polyclonal antiLC3 (Novus Biologicals, Littleton, CO, USA, NB100-2220, 1:2000 for IB); guinea pig polyclonal anti-p62 (American Research Product, Waltham, MA, USA, 03-GP62-C, 1:5000 for IB, 1:500 for IF, 1:800 for PLA); rabbit monoclonal anti-caspase-8 (Abcam, Cambridge, UK, ab108333, 1:600 for PLA); mouse monoclonal anti-Atg5 (MBL, Woburn, MA, USA, M153-3, 1:1000 for IB); goat polyclonal anti-Atg7 (Santa Cruz, Dallas, TX, USA, sc-8668, 1:300 for IB); rabbit polyclonal anti-Atg16L1 (MBL, PM040, 1:500 for IF); rabbit polyclonal anti-Atg2A (MBL, PD041, 1:1000 for IB); rabbit polyclonal anti-Atg2B (Sigma, HPA019665, 1:200 for IB); mouse monoclonal
}

anti-caspase-8 (Cell Signaling Technology, Danvers, MA, USA, 9746, 1:1000 for IB); rabbit monoclonal anti-cleaved caspase-8 (Cell Signaling Technology, 9496, 1:1000 for IB); rabbit monoclonal anti-cleaved caspase-3 (Cell Signaling Technology, 9664, 1:1000 for IB); rabbit polyclonal anti-PARP (Cell Signaling Technology, 9542, 1:1000 for IB); rabbit polyclonal anti-caspase-9 (Cell Signaling Technology, 9502, 1:1000 for IB); mouse monoclonal anti-FADD (BD Bioscience, San Jose, CA, USA, 556402, 1:500 for IB); mouse monoclonal anti- $\beta$-actin (Sigma, A5441-200UL, 1:10000 for IB); mouse monoclonal anti- $\alpha$-tubulin (Sigma, T5168-100UL, 1:4000 for IB); rabbit monoclonal anti-GFP (Abcam, ab32146 1:500 for immuno-EM). The following secondary antibodies have been used in this work: donkey anti-guinea pig IRDye 680RD (Licor, 925-68077); donkey anti-rabbit IRDye 680RD (Licor, Lincoln, NE, USA, 925-68073); donkey anti-rabbit IRDye 800CW (Licor, 925-32213), donkey antimouse IRDye 800CW (Licor, 925-32212); donkey anti-mouse IRDye 680RD (Licor, 925-68072); donkey anti-goat IRDye 800CW (Licor, 925-32214); goat anti-rabbit Alexa Fluor 488 (Life Technologies, Carlsbad, CA, USA, A11008); goat anti-rabbit Alexa Fluor 647 (Life Technologies, A21244); goat anti-rabbit Alexa Fluor 405 (Life Technologies, A31556); goat anti-guinea pig Alexa Fluor 488 (Life Technologies, A11073); goat anti-guinea pig Alexa Fluor 555 (Life Technologies, A21435); Nanogold anti-rabbit (Nanoprobes, Yaphank, NY, USA, 2004). Secondary antibodies were diluted 1:10 000 for IB, 1:2000 for IF, and 1:500 for immunogold TEM. The following flow cytometry antibodies/staining solution were used in this work: APC Annexin-V (Biolegend, San Diego, CA, USA, 640941) and 7-AAD (BD, BDB559925). The following constructs were used in this work: plenti-CRISPR v2 (Addgene, Cambridge, MA, USA, 52961); pLX-sgRNA (Addgene, 50662); LRG (Addgene, 65656); pLC-BFP-FADD (Addgene, 75166); pBiFC-c-FLIP-VN155 (1152L); pBiFC-caspase-8(C360A)-VN155(1152L) and pBiFC-caspase-8(C360A)VC155. ${ }^{13}$ mRFP-EGFP-LC3 (ptfLC3; Addgene, 21074) was subcloned into pCDH1MCS1-EF1-Puro vector using Nhell-EcoRl site. pCDH1-EGFP-Atg2 $A^{R}$ containing gRNA-resistant hAtg2A cDNA was generated by site directed mutagenesis. Silent mutations were introduced to the PAM sequence and the neighboring nucleotides in the gRNA-targeted region of the CDNA using a mutagenic primer (5'-GCCTG GACCAGCTCAGCTTAGACCTCTACAAGGGCAGCGTTGC-3') and the QuikChange XL II kit (Agilent, Santa Clara, CA, USA) per the manufacturer's instructions.

Cell culture and starvation. THP-1 (ATCC, Manassas, VA, USA, TIB-202), U-2 OS (ATCC HTB-96), and HeLa cells (ATCC, CCL2) were maintained at $37^{\circ} \mathrm{C}$ and $5 \% \mathrm{CO}_{2}$ in Iscove's Modified Dulbecco's Medium (IMDM for THP-1) and Dulbecco's Modified Eagle's Medium (DMEM for U-2 OS and HeLa), supplemented with $10 \%$ fetal bovine serum (FBS), $100 \mu \mathrm{g} / \mathrm{ml}$ of streptomycin, 100 units $/ \mathrm{ml}$ penicillin, and $250 \mathrm{ng} / \mathrm{ml}$ amphotericin B. For starvation, cells were washed once with PBS followed by incubation in serum and amino acid-free DMEM containing high glucose and sodium pyruvate (Wako Chemicals, Richmond, VA, USA, 048-33575).

CRISPR/Cas9 genome editing and siRNA knockdown. Single guide RNA (gRNA) targeting Atg2A (5'-CGCTGCCCTTGTACAGATCG-3'), Atg7 (5'-AA CTCCAATGTTAAGCGAGC-3'), and non-targeting (5'-GACCGGAACGATCTCGCG TA-3') were sub-cloned into pLenti-CRISPR-V2 (Addgene 52961); gRNA targeting Atg2B (5'-ATGGACTCCGAAAACGGCCA-3') was sub-cloned into pLX-sgRNA (Addgene 50662); gRNA targeting p62 (5'-TCAGGAGGCGCCCCGCAACA-3', $5^{\prime}$-CGCTACACAAGTCGTAGTCT-3', and 5'-AAGATCGCCTTGGAGTCCGA-3') and caspase-9 (5'-CAATCTTCTCGACCGACACA-3', 5'-TGGATGTCCTCGATCATA TG-3', and 5'-ACACCCAGACCAGTGGACAT-3') were sub-cloned into LRG (Addgene 65656). Lentiviral packaging in HEK293 cells and transduction were performed as described previously. ${ }^{13}$ CrAtg2A/B cells were generated by transducing cells with pLenti-CRISPR-Atg2A followed by 5 days of puromycin selection prior to transduction with $\mathrm{pLX}$-sgAtg2B lentivirus and blasticidin selection for 7 days. Single clones were isolated and screened for gene disruption by immunoblotting. LRG-casp-9, LRG-p62, and pLC-BFP-FADD (Addgene, 75166) were delivered by lentiviral transduction followed by FACS sorting. Edit-R human Atg5 (9474) crRNA and Edit-R human CASP-8 (841) crRNA were purchased from Dharmacon and transfected per manufacturer's instruction. Single clones were isolated and screened for gene disruption by immunoblotting. $200 \mathrm{pM}$ of OnTargetplus Human CFLAR (8837) siRNA-SMARTpool (Dharmacon, Lafayette, CO, USA, L-003772-00-0005) and OnTargetplus Human Non-Targeting siRNASMARTpool (Dharmacon, D-001810-10-05) were transfected into THP-1 cells to knockdown c-FLIP. 
Autophagy assays. Autophagy assays were performed as previously described. ${ }^{55}$ Briefly, cells were starved in the presence or absence of $100 \mathrm{nM}$ Bafilomycin A1 prior to immunoblotting. The intensity of LC3-II and $\beta$-actin were quantified using LI-COR Biosciences Image Studio 5.0 software, and LC3-II intensities were normalized to $\beta$-actin. Basal autophagic flux (1) and starvation-induced autophagic flux (2) were calculated using the following formulas: ${ }^{56}$ (1) [(complete media with BafA1 - complete media)/(complete media with BafA1)] $\times 100$; (2) [(starvation media with BafA1 - starvation media)/(starvation media with BafA1)] $\times 100$. All data were normalized to WT.

Immunoblotting and nuclear fractionation. Western blotting analyses were performed as described previously. ${ }^{55}$ Briefly, cells were harvested, washed once with ice-cold PBS and lysed with RIPA buffer containing protease (Sigma, P8340) and phosphatase (Sigma, P5726) inhibitors. Protein concentrations were quantified by BCA assay (ThermoFisher Scientific, 23225). Equal total protein was subjected to electrophoresis and transferred to PVDF membranes. Blots were developed using the LI-COR C-DiGit Blot Scanner or LI-COR Odyssey CLx. Nuclear fractionation was performed by using Qproteome Nuclear Protein Kit (Qiagen, Hilden, Germany, 37582) per manufacturer's instruction.

Microscopy. Fluorescence microscopy was performed as described previously. ${ }^{55}$ Briefly, THP-1 or U-2 OS cells were subjected to cytospin or seeded on Lab-Tekll Chamber Slides (Thermo Scientific, 154917), respectively. Cells were fixed in $4 \%$ paraformaldehyde-PBS, permeabilized with $100 \mu \mathrm{g} / \mathrm{ml}$ digitonin for $10 \mathrm{~min}$, blocked with $10 \%$ normal goat serum for $1 \mathrm{~h}$ and incubated with the indicated primary antibodies overnight. Secondary antibodies were incubated for $1 \mathrm{~h}$ at room temperature prior to mounting with ProLong Gold mounting medium with or without DAPI. Images were obtained using an Olympus IX81 inverted fluorescence microscope or Leica AOBS SP8 confocal microscope. Huygens, Imaris, and SlideBook softwares were used for image deconvolution and analyses. Immunogold labeling and transmission electron microscopy (TEM) were performed as described previously. ${ }^{55,57,58}$

Viability and apoptosis assays. Cell viability, caspase- 8 , and caspase- $3 / 7$ activities were measured using CellTiter-Glo (Promega, Madison, WI, USA), Caspase-Glo 8 (Promega), and Caspase-Glo 3/7 (Promega) assays per the manufacturer's instructions. For flow cytometry cell death analyses, treated cells were washed once with PBS and stained with $5 \%$ Annexin-V and 7-AAD for 15 min before flow cytometric analyses. All data were normalized to their non-treated controls.

Bimolecular fluorescence complementation and proximity ligation assays. BiFC was performed as described previously. ${ }^{13}$ Briefly, cells were transiently transfected with pBiFC-caspase-8(C360A)-VN155(1152L) and pBiFC-caspase-8(C360A)-VC155 or pBiFC-C-FLIP-VN155(1152L) and pBiFCcaspase-8(C360A)-VC155 using the Nucleofector Kit-V (Lonza, Basel, Switzerland, U-2 OS, program X-001) or FuGENE HD Transfection Reagent (Promega, E2311; U-2 OS) or Human Monocyte Nucleofector Kit (Lonza, THP-1, program Y-001) per the manufacturer's instructions. Cells were allowed to recover for $24 \mathrm{~h}$ before experimentation. After treatment, cells were fixed in $4 \%$ paraformaldehyde, immunostained where indicated and mounted with DAPI. BiFC signals were quantified using Slidebook 6.0 software. PLA antibody-oligo conjugation and PLA assays were performed per the manufacturer's instructions (Sigma, DUO920091KT, DU092010-1KT, and DU092014-30RXN).

Statistical analysis. Statistical significance was evaluated by unpaired two-tailed Student's $t$-test or ANOVA with multiple comparisons and Tukey post hoc analysis using GraphPad (La Jolla, CA, USA) Prism 7 software. Thresholds for significance are indicated on each figure.

\section{Conflict of Interest}

The authors declare no conflict of interest.

Acknowledgements. This work was supported by the Hyundai Hope on Wheels Foundation, Lois High Berstler, and Four Diamonds Fund. Authors would like to thank Dr. Kenichiro Doi, Neelam Desai, and Longgui Chen for technical support. We thank Nate Sheaffer, Joseph Bednarzyk, and Jade Vogel from the Penn State College of
Medicine Flow Cytometry Core for assistance with flow cytometry analyses. We would also like to thank Dr. Thomas Abraham and Wade Edris of the Penn State College of Medicine Imaging Core for assistance with the confocal microscope.

1. Mizushima N, Yoshimori T, Ohsumi Y. The role of Atg proteins in autophagosome formation Annu Rev Cell Dev Biol 2011; 27: 107-132.

2. Birgisdottir AB, Lamark T, Johansen T. The LIR motif - crucial for selective autophagy. J Cell Sci 2013; 126: 3237-3247.

3. Shintani T, Suzuki K, Kamada Y, Noda T, Ohsumi Y. Apg2p functions in autophagosome formation on the perivacuolar structure. J Biol Chem 2001; 276: 30452-30460.

4. Wang CW, Kim J, Huang WP, Abeliovich H, Stromhaug PE, Dunn WA Jr. et al. Apg2 is a novel protein required for the cytoplasm to vacuole targeting, autophagy, and pexophagy pathways. J Biol Chem 2001; 276: 30442-30451.

5. Kishi-Itakura C, Koyama-Honda I, Itakura E, Mizushima N. Ultrastructural analysis of autophagosome organization using mammalian autophagy-deficient cells. J Cell Sci 2014; 127: 4089-4102.

6. Velikkakath AK, Nishimura T, Oita E, Ishihara N, Mizushima N. Mammalian Atg2 proteins are essential for autophagosome formation and important for regulation of size and distribution of lipid droplets. Mol Biol Cell 2012; 23: 896-909.

7. Mariño G, Niso-Santano M, Baehrecke EH, Kroemer G. Self-consumption: the interplay of autophagy and apoptosis. Nat Rev Mol Cell Biol 2014; 15: 81-94.

8. Nelson DA, White E. Exploiting different ways to die. Genes Dev 2004; 18: 1223-1226.

9. Fulda S, Debatin KM. Extrinsic versus intrinsic apoptosis pathways in anticancer chemotherapy. Oncogene 2006; 25: 4798-4811.

10. Yang JK. Death effecter domain for the assembly of death-inducing signaling complex Apoptosis 2015; 20: 235-239.

11. Li H, Zhu H, Xu CJ, Yuan J. Cleavage of BID by caspase 8 mediates the mitochondrial damage in the Fas pathway of apoptosis. Cell 1998; 94: 491-501.

12. Tang D, Lahti JM, Kidd VJ. Caspase-8 activation and bid cleavage contribute to MCF7 cellular execution in a caspase-3-dependent manner during staurosporine-mediated apoptosis. J Biol Chem 2000; 275: 9303-9307.

13. Young MM, Takahashi $\mathrm{Y}$, Khan $\mathrm{O}$, Park S, Hori T, Yun $\mathrm{J}$ et al. Autophagosomal membrane serves as platform for intracellular death-inducing signaling complex (iDISC)-mediated caspase-8 activation and apoptosis. J Biol Chem 2012; 287: 12455-12468.

14. Bell BD, Leverrier S, Weist BM, Newton RH, Arechiga AF, Luhrs KA et al. FADD and caspase- 8 control the outcome of autophagic signaling in proliferating T cells. Proc Natl Acad Sci USA 2008; 105: 16677-16682.

15. Laussmann MA, Passante E, Dussmann H, Rauen JA, Wurstle ML, Delgado ME et al. Proteasome inhibition can induce an autophagy-dependent apical activation of caspase-8. Cell Death Differ 2011; 18: 1584-1597.

16. Pan JA, Ullman E, Dou Z, Zong WX. Inhibition of protein degradation induces apoptosis through a microtubule-associated protein 1 light chain 3-mediated activation of caspase-8 at intracellular membranes. Mol Cell Biol 2011; 31: 3158-3170.

17. Jiang H, White EJ, Rios-Vicil Cl, Xu J, Gomez-Manzano C, Fueyo J. Human adenovirus type 5 induces cell lysis through autophagy and autophagy-triggered caspase activity. $J$ Virol 2011; 85: 4720-4729.

18. Huang S, Okamoto K, Yu C, Sinicrope FA. p62/sequestosome-1 up-regulation promotes ABT-263-induced caspase-8 aggregation/activation on the autophagosome. J Biol Chem 2013; 288: 33654-33666.

19. Deegan S, Saveljeva S, Logue SE, Pakos-Zebrucka K, Gupta S, Vandenabeele P et al. Deficiency in the mitochondrial apoptotic pathway reveals the toxic potential of autophagy under ER stress conditions. Autophagy 2014; 10: 1921-1936.

20. Zhang YB, Gong JL, Xing TY, Zheng SP, Ding W. Autophagy protein p62/SQSTM1 is involved in HAMLET-induced cell death by modulating apotosis in U87MG cells. Cell Death Dis 2013; 4: e550.

21. Villar VH, Nguyen TL, Delcroix V, Teres S, Bouchecareilh M, Salin B et al. mTORC1 inhibition in cancer cells protects from glutaminolysis-mediated apoptosis during nutrient limitation. Nat Commun 2017; 8: 14124.

22. Pyo JO, Jang MH, Kwon YK, Lee HJ, Jun JI, Woo HN et al. Essential roles of Atg5 and FADD in autophagic cell death: dissection of autophagic cell death into vacuole formation and cell death. J Biol Chem 2005; 280: 20722-20729.

23. Jin Z, Li Y, Pitti R, Lawrence D, Pham VC, Lill JR et al. Cullin3-based polyubiquitination and p62-dependent aggregation of caspase-8 mediate extrinsic apoptosis signaling. Cell 2009; 137: 721-735.

24. Tazzari PL, Tabellini G, Ricci F, Papa V, Bortul R, Chiarini F et al. Synergistic proapoptotic activity of recombinant TRAIL plus the Akt inhibitor Perifosine in acute myelogenous leukemia cells. Cancer Res 2008; 68: 9394-9403.

25. Lee SY, Cherla RP, Caliskan I, Tesh VL. Shiga toxin 1 induces apoptosis in the human myelogenous leukemia cell line THP-1 by a caspase-8-dependent, tumor necrosis factor receptor-independent mechanism. Infect Immunity 2005; 73: 5115-5126.

26. Klionsky DJ, Abdelmohsen K, Abe A, Abedin MJ, Abeliovich H, Acevedo Arozena A et al. Guidelines for the use and interpretation of assays for monitoring autophagy (3rd edition). Autophagy 2016; 12: 1-222. 
27. Kimura S, Noda T, Yoshimori T. Dissection of the autophagosome maturation process by a novel reporter protein, tandem fluorescent-tagged LC3. Autophagy 2007; 3 : $452-460$.

28. Mizushima N, Yamamoto A, Hatano M, Kobayashi Y, Kabeya Y, Suzuki K et al. Dissection of autophagosome formation using Apg5-deficient mouse embryonic stem cells. J Cell Biol 2001; 152: 657-668.

29. Tsukada M, Ohsumi Y. Isolation and characterization of autophagy-defective mutants of Saccharomyces cerevisiae. FEBS Lett 1993; 333: 169-174.

30. Scott RC, Schuldiner O, Neufeld TP. Role and regulation of starvation-induced autophagy in the Drosophila fat body. Dev Cell 2004; 7: 167-178.

31. Kuma A, Hatano M, Matsui M, Yamamoto A, Nakaya $H$, Yoshimori $T$ et al. The role of autophagy during the early neonatal starvation period. Nature 2004; 432: 1032-1036.

32. Jin S, White E. Role of autophagy in cancer: management of metabolic stress. Autophagy 2007; 3: 28-31.

33. Kodama $\mathrm{Y}, \mathrm{Hu} \mathrm{CD}$. An improved bimolecular fluorescence complementation assay with a high signal-to-noise ratio. BioTechniques 2010; 49: 793-805.

34. Soderberg O, Leuchowius KJ, Gullberg M, Jarvius M, Weibrecht I, Larsson LG et al. Characterizing proteins and their interactions in cells and tissues using the in situ proximity ligation assay. Methods 2008; 45: 227-232.

35. Sanz L, Diaz-Meco MT, Nakano H, Moscat J. The atypical PKC-interacting protein p62 channels NF-kappaB activation by the IL-1-TRAF6 pathway. EMBO J 2000; 19 : 1576-1586.

36. Wooten MW, Geetha T, Seibenhener ML, Babu JR, Diaz-Meco MT, Moscat J. The p62 scaffold regulates nerve growth factor-induced NF-kappaB activation by influencing TRAF6 polyubiquitination. J Biol Chem 2005; 280: 35625-35629.

37. Duran A, Linares JF, Galvez AS, Wikenheiser K, Flores JM, Diaz-Meco MT et al. The signaling adaptor p62 is an important NF-kappaB mediator in tumorigenesis. Cancer Cell 2008; 13: 343-354.

38. Yang S, Qiang L, Sample A, Shah P, He YY. NF-kappaB signaling activation induced by chloroquine requires autophagosome, p62 protein, and c-Jun N-terminal kinase (JNK) signaling and promotes tumor cell resistance. J Biol Chem 2017; 292: 3379-3388.

39. Mathew R, Karp CM, Beaudoin B, Vuong N, Chen G, Chen HY et al. Autophagy suppresses tumorigenesis through elimination of p62. Cell 2009; 137: 1062-1075.

40. Tsuchiya Y, Nakabayashi O, Nakano H. FLIP the switch: regulation of apoptosis and necroptosis by cFLIP. Int J Mol Sci 2015; 16: 30321-30341.

41. Riley JS, Malik A, Holohan C, Longley DB. DED or alive: assembly and regulation of the death effector domain complexes. Cell Death Dis 2015; 6: e1866.

42. Kreuz $S$, Siegmund D, Scheurich $P$, Wajant $H$. NF-kappaB inducers upregulate cFLIP, a cycloheximide-sensitive inhibitor of death receptor signaling. Mol Cell Biol 2001; 21 : 3964-3973.
43. Micheau O, Lens S, Gaide O, Alevizopoulos K, Tschopp J. NF-kappaB signals induce the expression of c-FLIP. Mol Cell Biol 2001; 21: 5299-5305.

44. Lazova R, Camp RL, Klump V, Siddiqui SF, Amaravadi RK, Pawelek JM. Punctate LC3B expression is a common feature of solid tumors and associated with proliferation, metastasis, and poor outcome. Clin Cancer Res 2012; 18: 370-379.

45. Zhi X. Zhong Q. Autophagy in cancer. F1000prime Rep 2015; 7: 18

46. Fang J, Rhyasen G, Bolanos L, Rasch C, Varney M, Wunderlich M et al. Cytotoxic effects of bortezomib in myelodysplastic syndrome/acute myeloid leukemia depend on autophagymediated lysosomal degradation of TRAF6 and repression of PSMA1. Blood 2012; 120: 858-867.

47. Basit F, Cristofanon S, Fulda S. Obatoclax (GX15-070) triggers necroptosis by promoting the assembly of the necrosome on autophagosomal membranes. Cell Death Differ 2013; 20: 1161-1173.

48. Goodall ML, Fitzwalter BE, Zahedi S, Wu M, Rodriguez D, Mulcahy-Levy JM et al. The autophagy machinery controls cell death switching between apoptosis and necroptosis. Dev Cell 2016; 37: 337-349.

49. Martinez-Lopez N, Athonvarangkul D, Mishall P, Sahu S, Singh R. Autophagy proteins regulate ERK phosphorylation. Nat Commun 2013; 4: 2799.

50. Lin Y, Devin A, Rodriguez Y, Liu ZG. Cleavage of the death domain kinase RIP by caspase-8 prompts TNF-induced apoptosis. Genes Dev 1999; 13: 2514-2526.

51. Kwon SI, Cho HJ, Jung JH, Yoshimoto K, Shirasu K, Park OK. The Rab GTPase RabG3b functions in autophagy and contributes to tracheary element differentiation in Arabidopsis. Plant J 2010; 64: 151-164.

52. Lee $\mathrm{CY}$, Baehrecke EH. Steroid regulation of autophagic programmed cell death during development. Development 2001; 128: 1443-1455.

53. Lee CY, Cooksey BA, Baehrecke EH. Steroid regulation of midgut cell death during Drosophila development. Dev Biol 2002; 250: 101-111.

54. Berry DL, Baehrecke EH. Growth arrest and autophagy are required for salivary gland cell degradation in Drosophila. Cell 2007; 131: 1137-1148.

55. Takahashi Y, Tsotakos N, Liu Y, Young MM, Serfass J, Tang Z et al. The Bif-1-Dynamin 2 membrane fission machinery regulates Atg9-containing vesicle generation at the Rab11-positive reservoirs. Oncotarget 2016; 7: 20855-20868.

56. Tooze SA, Dooley HC, Jefferies HB, Joachim J, Judith D, Lamb CA et al. Assessing mammalian autophagy. Methods Mol Biol 2015; 1270: 155-165.

57. Takahashi Y, Coppola D, Matsushita N, Cualing HD, Sun M, Sato Y et al. Bif-1 interacts with Beclin 1 through UVRAG and regulates autophagy and tumorigenesis. Nat Cell Biol 2007; 9: $1142-1151$.

58. Takahashi Y, Hori T, Cooper TK, Liao J, Desai N, Serfass JM et al. Bif-1 haploinsufficiency promotes chromosomal instability and accelerates Myc-driven lymphomagenesis via suppression of mitophagy. Blood 2013; 121: 1622-1632.

Supplementary Information accompanies this paper on Cell Death and Differentiation website (http://www.nature.com/cdd) 